\title{
1 Compartmentalized antimicrobial defenses in response to flagellin
}

4 Aneesh VIJAYAN ${ }^{1}$, Martin RUMBO ${ }^{2}$, Christophe CARNOY ${ }^{1 \#}$, and Jean-Claude SIRARD ${ }^{1 \#}$

$6 \quad{ }^{1}$ Univ. Lille, CNRS, Inserm, CHU Lille, Institut Pasteur de Lille, U1019 - UMR8204 - CIIL -

7 Center for Infection and Immunity of Lille, F-59000 Lille, France

$8{ }^{2}$ Instituto de Estudios Inmunológicos y Fisiopatológicos - CONICET- National Universtity of

9 La Plata, 1900 La Plata, Argentina

10

11

12 \# Correspondence: christophe.carnoy@univ-lille2.fr; jean-claude.sirard@inserm.fr

13 
14 Motility is often a pathogenicity determinant of bacteria targeting mucosal tissues. Flagella

15 constitute the machinery that propels bacteria into appropriate niches. Besides motility, the

16 structural component flagellin, which forms flagella, targets Toll-like receptor 5 (TLR5) to

17 activate innate immunity. The compartmentalization of flagellin-mediated immunity and the

18 contribution of epithelial cells, and dendritic cells in detecting flagellin within luminal and basal

19 sides are highlighted here, respectively. While a direct stimulation of the epithelium mainly

20 results in recruitment of immune cells and production of antimicrobial molecules, TLR5

21 engagement on parenchymal dendritic cells can contribute to the stimulation of innate

22 lymphocytes such as type 3 innate lymphoid cells, as well as $\mathrm{T}$ helper cells. This review,

23 therefore, illustrates how the innate and adaptive immunity to flagellin are differentially

24 regulated by the epithelium and the dendritic cells in response to pathogens that either colonize

25 or invade mucosa.

26 (143 words) 


\section{Detection of bacterial flagellin and regulation of immune responses}

Bacterial infection of mammalian hosts triggers pro-inflammatory responses, i.e., innate

29 immune responses, through detection of microbe-associated molecular patterns (MAMP) by

30 pattern recognition molecules (PRM). MAMPs are conserved molecules of microorganisms that

31 bind to PRM initiating a signaling cascade that culminates in the pro-inflammatory response. The

32 main feature of these events involves the activation of sentinel cells that are the cornerstone of

33 innate and adaptive antibacterial defenses. This review describes how flagellins, significant

34 MAMPs of flagellated bacteria, contribute to immunity with emphasis on the mucosal tissues

35 and compartmentalization of responses.

\section{Structural organization of flagellum and flagellin}

Flagellin is the structural protein of flagellum, a surface filament dedicated to bacterial

39 motility. The filament can be formed of as many as 20,000 subunits of flagellin. In pathogenic

40 bacteria, flagella and the chemotaxis machinery can contribute to virulence [1]. The amino-acid

41 composition of flagellin ranges between 250 to 1250 residues that include a conserved region

42 flanking a central hypervariable region. The 170 residues at the $\mathrm{N}$-terminal end and 100 residues

43 at the C-terminal end are highly conserved sequences among different genera of bacteria. The

44 structure of Salmonella enterica serovar Typhimurium flagellin FliC, a 495 amino-acid long

45 protein, has been defined (Figure 1A). The terminal chains that form the packed helical

46 structures, i.e., the D0 and D1 domains, are essential for polymerization of flagellin into the

47 flagellum. The central hypervariable region (i.e., the D2 and D3 domains) that determines the

48 flagellin serovar ( $\mathrm{H}$ typing) is exposed as beta-sheet and turn-folded structures on the outer

49 surface of the filament [2]. Thus, the D2 and D3 sequences are under intense selective pressure 
50 and vary to escape the protective/neutralizing antibody responses (through phase variation for

51 example), thus allowing colonization and infection by new bacterial serotypes. All domains are

52 compactly folded in flagellum whereas the D0 domain is disordered in solution, i.e., when

53 flagellin is released as free monomers [3].

55 Detection by pattern-recognition molecules

Toll-like receptor 5 (TLR5), a mammalian PRM, senses extracellular flagellin monomers

57 [4]. Orthologues of TLR5 are distributed among vertebrates and invertebrates in addition to the

58 so-called flagellin-sensitive two receptor or FLS2, a TLR5-like PRM, in plants (Box 1). Both

59 human and mouse TLR5 are type I transmembrane proteins that recognize similar molecular

60 determinants of native flagellin and have a comparable effective concentration in cell-based

61 assays [5]. While the hypervariable part of the $S$. Typhimurium flagellin FliC is dispensable to

62 TLR5 signaling, the D1 domain, especially the conserved residues 89-96 (QRIRELAV), as well

63 as the D0 domain, are essential [6-10]. Most epsilon-and alpha-proteobacteria produce flagellins

64 with an alternative 89-96 motif (such as DTVKVKAT or DTIKTKAT) that does not activate

65 TLR5 and evades immune sensing. The $89-96$ motif that is embedded in the flagellum core is

66 accessible to PRM only when flagellin is released as a monomer (Figure 1B-C). The

67 extracellular domain of TLR5 is composed of 22 leucine-rich repeats (LRR). Mutagenesis and

68 crystallographic studies (based on the zebrafish TLR5 and flagellin from S. enterica serovar

69 Dublin) provided clues on the receptor interaction with flagellin [9]. This model was validated

70 functionally on mammalian TLR5. Two regions of flagellin are essential for primary interaction

71 with TLR5 that lead to the establishment of TLR5-FliC 1:1 heterodimer: (1) the carboxy-

72 terminal $\alpha$-helix of the D1 domain that is in close contact with the TLR5 LRRs 1-6, and (2) the 
motif $89-86$ of the D1 domain that binds to LRR7-10. Moreover, LRRs 12-13 promotes the

74 dimerization of TLR5-FliC 1:1 heterodimer to build up a 2:2 functional complex (Figure 1C).

75 Interestingly, deletion of the C-terminal region (from LRR14 to C-terminal) results in a secreted

76 and soluble TLR5 form that still binds flagellin (Box 2). While the D0 domain of flagellin is

77 essential to TLR5 signaling the molecular interactions between D0 and TLR5 are not entirely

78 understood [10]. A recent study demonstrated that the C-terminal part of the D0 domain of

79 flagellin is essential to TLR5 signaling whereas the N-terminal one is dispensable [11]. The

80 dissection of molecular mechanisms of D0 and TLR5 will be of major interest to unravel how

81 interaction is integrated into the activation of signaling cascade.

Recently, the high mobility group box 1 (HMGB1), an alarmin released from necrotic

83 cells, has been found to depend on TLR5 signaling [12]. Even though it was determined that the

84 carboxy-terminal region of HMGB1 is involved in TLR5 interaction, further studies will be

85 required to understand the molecular mechanisms involved.

TLR5 is ubiquitously expressed at the surface of various cells including myeloid cells

87 (macrophages, dendritic cells [DC], or monocytes), lymphoid cells (lymphocytes, or natural

88 killer cells), structural cells (epithelial cells [EC] from mucosa, keratinocytes, or fibroblasts) or

89 neurons (reviewed in $[13,14]$ ). It is important to stress out that TLR5 expression has mainly been

90 studied by RT-qPCR and knockout models but has not been validated at the protein levels due to

91 the lack of quality controlled TLR5-specific antibodies. Interestingly, the Unc-93 homolog B1 of

92 Caenorhabditis elegans (UNC93B1), an essential protein for intracellular traffic of PRM,

93 promote the addressing of TLR5 to the plasma membrane, thereby regulating TLR5 signaling

94 [15]. TLR5 signaling requires the myeloid differentiation primary response 88 (MyD88), an

95 adaptor protein essential for signaling by most TLRs as well as interleukin receptors including 
96 IL-1R, IL-18R, IL-33R, and IL-36R (Figure 1B) [4,16]. TLR5 and MyD88 assemble into a high

97 scaffold-signaling complex (the so-called myddosome) that ultimately activates nuclear factor

98 (NF)- $\mathrm{kB}$, mitogen-activated protein kinase (MAPK), and interferon (IFN)-regulatory factor

99 (IRF) pathways [17]. This cascade upregulates transcription of genes coding for immune

100 mediators as described later in this review. The flagellin-mediated response is short-lived due to

101 the strong feedback regulatory mechanisms involving transcriptional and post-transcriptional

102 regulators such as tumor necrosis factor alpha-induced protein 3 or TNFAIP3, mRNA decay

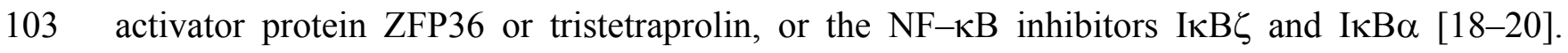

104 Several studies in TLR5-deficient animals stressed on flagellin's role in antibacterial defenses

105 [21-23]. Thus, TLR5 signaling is associated with the regulation of cytokine/chemokine

106 synthesis, the recruitment of phagocytes such as neutrophils, and the production of antimicrobial

107 molecules.

108 TLR11 was proposed to sense flagellin in the gut using the same motifs as TLR5 that in

109 turn protect against pathogenic intestinal bacteria in mice [24]. While TLR11 is not functional in

110 humans, in mice, it was initially shown to detect uropathogenic Escherichia coli MAMPs in

111 kidneys as well as the Toxoplasma gondii protein profilin $[25,26]$. Remarkably, TLR5 and

112 TLR11 belong to different phylogenic families in contrast to TLRs that perceive related

113 compounds like nucleic acids [27]. Recent findings pointed out that flagellin-mediated

114 stimulation of immunity and antimicrobial defenses do not require TLR11 signaling [28].

115 Moreover, most observations in TLR5-deficient animals support that TLR11 cannot substitute

116 for TLR5 and, therefore, is not required for immunostimulatory activity of flagellin in various

117 mouse tissues including the gut. Taken as a whole, TLR11 is unlikely to be involved in detecting

118 flagellin. 
Finally, bacterial flagellin is a unique MAMP, in the sense that it can trigger immune

120 signaling in the extracellular medium via TLR5 as well as in the cytoplasmic compartment. The

121 cytosolic detection of flagellin, at least in mice, is associated with the triggering of the

122 inflammasome (reviewed in [29]) (Box 3).

124 Epithelial responses to flagellin at mucosal surfaces

Apical detection of the bacterial danger signal is the first event that explains how

126 flagellated bacteria are sensed in the lumen by the impermeable mucosa in the absence of any

127 injury. At the mucosal surfaces, ECs are the first cells that sense flagellin and modulate immune

128 responses. Evidence from invertebrate species indicates that TLR-mediated flagellin recognition

129 at epithelial surfaces is an old feature that has its evolutionary origin in the appearance of a stable

130 association of microbiota to metazoans (Box 1). Intestinal, respiratory, and urogenital ECs, as

131 well as keratinocytes derived from cell lines or primary cultures, are highly responsive to

132 flagellin stimulation through TLR5 (reviewed in $[13,14]$ ). Initially, the interaction of flagellin

133 with TLR5 was proposed to be exclusively at the basolateral compartment of the epithelial layer

134 as exemplified by polarized human intestinal cell line T84 [30]. Currently, there is evidence that

135 TLR5 is also expressed at the apical pole of ECs from the follicle-associated epithelium (FAE)

136 of Peyer's patches, the small intestine or the respiratory mucosa [18,31-34].

137 Flagellin-stimulated ECs rapidly induce pro-inflammatory mediators such as chemokines

138 CXCL1, CXCL2, CXCL5, CXCL8, CCL2, CCL20 or CXCL10 that in turn attract immune cells 139 belonging to the myeloid and lymphoid origin (reviewed in $[13,14]$ ). This process is essential for

140 recruiting cells that participate in innate response, thereby shaping antimicrobial defenses against

141 flagellated bacteria (Figure 2). For instance, in wild-type animals that were reconstituted with 
$142 \mathrm{Tlr}^{-/}$bone marrow, radioresistant cells, mainly respiratory ECs produce chemokines in response 143 to flagellin, leading to the infiltration of polymorphonuclear neutrophils (PMNs) in the 144 conducting airways [18,35,36]. Moreover, TLR5 signaling elicits production of cytokines and 145 cell growth factors (such as IL-6, IL-1 $\beta$, granulocyte colony-stimulating factor or G-CSF) that 146 further contribute to activation and survival of recruited cells. Finally, stimulation of ECs by 147 flagellin is characterized by the polarized secretion of various antimicrobial peptides or 148 molecules including mucins and $\beta$-defensins in the mucosal lumen that can contribute to host 149 defense (reviewed in $[13,14])$. Using mice deficient for MyD88 in lung epithelial cells, flagellin150 deficient, and proficient bacteria, and $T l r 5^{-/}$bone marrow chimera, a recent study further 151 highlights the role of ECs in orchestrating TLR5-dependent innate defenses against bacterial 152 respiratory infections [35].

153 TLR5-dependent epithelium signaling also impacts the adaptive response by regulating 154 antigen transport in the lamina propria of mucosa and DC activation. First, TLR5 signaling in 155 FAE facilitates transportation and antigen delivery to immature DCs via M cells [37]. 156 Remarkably, the chemokine CCL20, a ligand for $\mathrm{CCR}^{+} \mathrm{DCs}$, is constitutively expressed by 157 FAE and can be upregulated by flagellin in a TLR5-dependent manner [32,38]. Upon maturation, 158 by danger signals including MAMPs or by cytokines, DCs migrate to draining lymphoid tissues, 159 present antigens to naïve lymphocytes and polarize the adaptive responses. Indeed, DCs provide 160 appropriate signals for the development of $\mathrm{CD}^{+} \mathrm{T}$ helper cell (Th), regulatory $\mathrm{T}$ cell (Treg), $161 \mathrm{CD}^{+} \mathrm{T}$ cell, and B cell responses. Immature DCs in tissues are mostly conventional DCs (cDCs) 162 expressing CD103 (cDC1) or CD11b/CD172 $\alpha$ (cDC2) [39]. Flagellin administration by the 163 respiratory route induces, via $\mathrm{ECs}$, the maturation of $\mathrm{cDC} 1$ and $\mathrm{cDC} 2$, as monitored by 164 upregulation of MHCII, co-stimulatory molecules and antigen presentation [19,20]. The nature 
165 of EC signals that contribute to DC maturation remains to be defined. This indirect effect on the

$166 \mathrm{cDC}$ is essential for the TLR5-dependent mucosal adjuvant effect of flagellin. Indeed, the

167 flagellin-mediated mucosal adjuvant effect on antigen-specific $\mathrm{CD}^{+}{ }^{+} \mathrm{T}$-cell responses is a 168 process dependent on $\mathrm{cDC} 2$ [20]. Garont et al. recently demonstrated that human cDC2

169 preferentially home to mucosal tissues, suggesting that these cells can be a prime target for 170 mucosal adjuvants [40]. In conclusion, flagellin is an efficient mucosal adjuvant for nasal, 171 respiratory and oral vaccinations [41,42]. The application of flagellin by mucosal route is 172 associated with mixed Th1 and Th2 responses, systemic IgG1 production, and secretory $\operatorname{IgA}$ in 173 the mucosa lumen. Interestingly, IgA class switching in gut B cells is partly mediated by 174 flagellin-dependent secretion of a proliferation-inducing ligand (APRIL) by ECs [43].

175 Respiratory administration of flagellin was suggested to be associated with the priming of allergy 176 [44]. However, a recent study demonstrated that flagellin downregulates allergic response 177 [45].This discrepancy may be due to the quantity and purity of flagellin that allows the 178 development of Tregs involved in the desensitization to allergens.

179 Taken together, the sensing of flagellin by ECs is a major process for shaping the 180 immune response against flagellin and flagellated bacteria.

182 Direct activation of dendritic cells: key mechanism of systemic response to flagellin

183 Innate immune sensors, such as TLRs, are essential for the direct activation of innate and 184 adaptive immunity by DCs. This section discusses the TLR5-mediated effect of flagellin 185 monomer on DC and in turn on adaptive immunity (Figure 3). This mode of action can have a 186 significant impact when flagellated bacteria invade mucosal tissues and deliver flagellin in the 187 parenchyma compartment or deeper tissues. 
Flagellin was initially reported to stimulate Th1-type responses when administered by 189 systemic route [46,47]. However, our observations and studies from Cunningham et al. also 190 support induction of Th2 responses against $S$. Typhimurium flagellin [16,48]. Thus, flagellin 191 promotes the development of TLR5- and MyD88-dependent mixed Th1-Th2 responses when co192 administered with protein antigens. It is characterized by the production of interferon- $\gamma$, IL-4, 193 and IL-13 by antigen-specific $\mathrm{CD}^{+} \mathrm{T}$ lymphocytes, and prominent IgG1 antibody isotype. 194 Indeed, systemic administration of flagellin induces DC maturation and up-regulation of co195 stimulatory molecules and antigen-presenting capacity in mucosal tissues as well as in secondary 196 lymphoid tissues such as the spleen or lymph nodes (Figure 3). Notably, DCs are a primary 197 source of TLR5 within the myeloid compartment. In DCs from mouse or human, flagellin 198 triggers secretion of IL-12p40 (but not IL-12p70), that is pivotal for instructing and maintaining 199 Th2 cells and to a certain extent Th1 cells [49,50]. Recently, gut cDC2 cells were found to 200 respond to flagellin by producing IL-23 (an heterodimer of IL-12p40 and IL-23p19), IL-6, and 201 Transforming Growth Factor $\beta$ (TGF $\beta$ ) [51-54]. This TLR5-dependent activity led to the 202 induction of the Th17-type cytokine IL-22 by the type 3 innate lymphoid cells (ILC3) and the 203 development of Th17 responses [19,51,53-55]. In contrast to the lymphocyte activation in 204 lymphoid tissue due to migratory DCs, ILC3 activation is immediate and depends heavily on 205 tissue-resident DCs. Moreover, CD103 ${ }^{+}$DCs (likely cDC1s) recruited to mesenteric lymph 206 nodes, upon systemic flagellin administration, can prime Treg expressing FoxP3 and IgA207 producing B cells [56]. How different subsets of cDCs that are stimulated by flagellin/TLR5 208 influence innate and adaptive immunity remain to be investigated with regards to the route of 209 administration, the amount of flagellin, and the mucosal tissue environment (see Outstanding 210 Questions). 


\section{Manipulation of flagellin-specific signaling for prophylaxis or treatment of diseases}

213 The ability of flagellin to elicit a prompt and robust innate response that in turn primes

214 the adaptive arm of the immune system makes it attractive as an immunomodulatory adjuvant for

215 control of various diseases. Studies in animals and non-human primates show flagellin as an

216 effective vaccine adjuvant when administered with an antigen or produced as a fusion protein

217 with antigen [41,42,57]. Interestingly, the potency of flagellin to induce strong adaptive immune

218 responses, in a low-dose regimen, is appealing for flagellin-based vaccines. Recombinant

219 flagellins derived from Salmonella species have already been tested in some clinical trials that

220 are ongoing or are completed (for review see: clinicaltrials.gov). The safety of recombinant

221 flagellins, as well as their capacity to promote an immuno-adjuvant effect, was validated in

222 human volunteers using intramuscular or subcutaneous routes of administration with the

223 effective doses of flagellin ranging from $1 \mathrm{ng} / \mathrm{kg}$ to $300 \mathrm{ng} / \mathrm{kg}$. Furthermore, the adjuvant

224 properties of flagellin have been demonstrated in the context of influenza vaccine candidates in

225 neonatal non-human primates and clinical studies with older subjects, indicating that the

226 efficiency of flagellin is independent of the age $[58,59]$. The flagellin's adjuvant activity is

227 extensively associated with TLR5 signaling (Box 2 and Box 3). However, some studies suggest

228 that neither TLR5 nor NAIP5/6 is required for immune signaling, but a pathway that remains to

229 be identified [60-62]; The dose and the quality of flagellin, as well as the route of administration,

230 might explain this discrepancy. Further studies are still required to evaluate flagellin's crosstalk

231 with the immune system and how this, in turn, influences its adjuvant activity.

232 TLR5-deficient mice are more susceptible to intestinal, respiratory or urinary tract

233 bacterial infection, indicating the central role of flagellin in innate antibacterial defenses [21-23]. 
234 Flagellin-based interventions have demonstrated protective activity in many infectious diseases.

235 Mucosal or systemic administration of standalone flagellin protects mice against intestinal, 236 respiratory or cutaneous infections caused by either Gram-negative (Salmonella sp.,

237 Pseudomonas aeruginosa, Burkholderia cepacia, Yersinia pseudotuberculosis) or Gram-positive

238 bacteria (Enterococcus faecalis, Clostridium difficile, or Streptococcus pneumoniae) [63-69].

239 The antibacterial effects of flagellin correlate with the epithelial production of antimicrobial

240 compounds such as RegIII $\gamma$, cathelicidin, $\beta$-defensin-2 or calgranulins [64,67,70-73] (Figure 2).

241 The protection also depends on the recruitment of neutrophils in the mucosal tissues through

242 TLR5-dependent activation of ECs or the DC/ILC3 axis. The contribution of the epithelial

243 compartment to neutrophil infiltration was supported by recent studies $[35,69,74]$. Protective

244 effects of flagellin treatment were mainly defined in the prophylactic regimen, i.e., when

245 administered prior or at the time of infection. Interestingly, the combination of flagellin with

246 antibiotics was very effective for therapeutic intervention against acute bacterial respiratory

247 infection [74]. Such combinatory treatments were more effective than standalone antibiotic

248 therapy in post-influenza pneumococcal infection, a pathophysiological context where the lung is

249 actively inflamed and infected, and tissue architecture is altered [74].

250 Flagellin is also reported to promote antiviral activity. When delivered $48 \mathrm{~h}$ to $72 \mathrm{~h}$ before

251 challenge, the TLR5 agonist CBLB502 or Entolimod (a recombinant truncated flagellin from $S$.

252 enterica serovar Dublin) protects mice from lethal infection by cytomegalovirus in an NK cell-

253 dependent manner [75]. Flagellin also protects against intestinal viral infection as daily systemic

254 administration of flagellin prevents mice from rotavirus infection and cures chronically infected 255 mice $[63,76]$. The resistance mediated by flagellin administration requires TLR5 and NLRC4, 
256 which are responsible for the production of IL-22 and IL-18, respectively [76]. Combined 257 administration of both IL-22 and IL-18 recapitulates antiviral defense.

258 The therapeutic effect of flagellin goes beyond protection against infections. Indeed, a single 259 dose of Entolimod, administrated before lethal radiation, improves the survival probability of 260 mice and non-human primates [63,77]. Flagellin induces irradiation resistance of the 261 radiosensitive intestinal mucosa through $\mathrm{NF}-\mathrm{kB}$-mediated anti-apoptotic properties. The liver is 262 an essential target of flagellin treatment, participating in Entolimod-mediated radioprotection; 263 However, the molecular and cellular mechanisms remain unknown [78]. Flagellin also 264 demonstrated efficacy in experimental cancer prevention either by a direct effect on TLR5265 expressing tumor cells or by activating bystander immune and non-immune cells [78-80]. For 266 instance, tumor-specific $\mathrm{T}$ cells engineered to produce flagellin contributes to antitumor efficacy 267 by increasing $\mathrm{T}$ cell infiltration and reducing the recruitment/activation of Treg cells and 268 myeloid-derived suppressor cells [81]. More recently, injection of attenuated $S$. Typhimurium 269 designed to secrete heterologous flagellin decreases tumor volume and improves survival of mice 270 through tumor-suppressor M1-type macrophages [82]. Altogether, flagellin via TLR5 signaling 271 provides a unique opportunity to manipulate immunity for vaccine adjuvants, infection 272 treatment, radioprotective countermeasures, or cancer immunotherapy. 


\section{Concluding remarks}

275 Given the nature of the mucosa and its continuous exposure to antigens and pathogens,

276 the epithelial cells, that constitute the mucosa, are more than physical barriers between the

277 internal and external environment. Because they express PRM, ECs cells represent sentinel cells

278 able to sense danger signals such as MAMPs on the outer side. In contrast, when inside MAMPs

279 directly impact the PRM signaling in mucosal myeloid cells. The immune responses induced by

280 TLR5 and flagellin interaction in mucosa are profoundly influenced by the nature of sentinel

281 cells. ECs and cDCs similarly turn on TLR5 signaling but differentially regulate the set of

282 immune mediators expressed in luminal and tissue contexts and therefore compartmentalize the

283 immune responses. Flagellin-stimulated ECs mainly produce chemokines and cytokines to

284 promote recruitment and activation of myeloid cells, including neutrophils and cDCs. In contrast,

285 direct stimulation of tissue-resident cDCs by flagellin transactivates the lymphoid components

286 including the type 3 innate lymphoid cells. Given the central role that DCs play in shaping the

287 adaptive responses, a direct or indirect action of flagellin on these cells holds great promise to

288 understand the evolution of acquired immunity against mucosal microbial infections. In

289 conclusion, formulating MAMPs, like flagellin, that specifically target mucosal compartments or

290 sentinel cells offers the prospect of developing novel PRM-mediated host-directed therapies. 


\section{Acknowledgments}

294 AV is supported by a Marie Sklodowska-Curie Actions Individual Fellowship from the European 295 Commission (H2020-MSCA-IF-2014 Grant Agreement No. 657107). The Argentinean National 296 Research Council (CONICET) supports MR. AV, JCS, and CC are supported by the Institut 297 Pasteur de Lille, Inserm, CNRS, the Université de Lille, the national fund agency ANR (ANR298 16-ASTR-0013-01) and the nonprofit organization Vaincre la Mucoviscidose 299 (RF20160501644/1/1/86).

300 


\section{References}

3021 Chaban, B. et al. (2015) The flagellum in bacterial pathogens: For motility and a whole lot 303 more. Semin. Cell Dev. Biol. 46, 91-103

3042 Yonekura, K. et al. (2003) Complete atomic model of the bacterial flagellar filament by 305 electron cryomicroscopy. Nature 424, 643-50

3063 Aizawa, S.-I. et al. (1990) Termini of Salmonella flagellin are disordered and become 307 organized upon polymerization into flagellar filament. J. Mol. Biol. 211, 673-7

3084 Hayashi, F. et al. (2001) The innate immune response to bacterial flagellin is mediated by 309 Toll-like receptor 5. Nature 410, 1099-1103

3105 Forstnerič, V. et al. (2016) Distinctive Recognition of Flagellin by Human and Mouse 311 Toll-Like Receptor 5. PLoS One 11, e0158894

3126 Andersen-Nissen, E. et al. (2005) Evasion of Toll-like receptor 5 by flagellated bacteria. $313 \quad$ Proc. Natl. Acad. Sci. 102, 9247-52

3147 Nempont, C. et al. (2008) Deletion of flagellin's hypervariable region abrogates antibody315 mediated neutralization and systemic activation of TLR5-dependent immunity. $J$. $316 \quad$ Immunol. 181, 2036-43

3178 Smith, K.D. et al. (2003) Toll-like receptor 5 recognizes a conserved site on flagellin 318 required for protofilament formation and bacterial motility. Nat. Immunol. 4, 1247-53

3199 Yoon, S. et al. (2012) Structural basis of TLR5-flagellin recognition and signaling. $320 \quad$ Science $335,859-64$

32110 Song, W.S. et al. (2017) A conserved TLR5 binding and activation hot spot on flagellin. 322 Sci. Rep. 7, 40878

32311 Forstnerič, V. et al. (2017) The role of the C-terminal D0 domain of flagellin in activation 
of Toll like receptor 5. PLOS Pathog. 13, e1006574

32512 Das, N. et al. (2016) HMGB1 Activates Proinflammatory Signaling via TLR5 Leading to $326 \quad$ Allodynia. Cell Rep. 17, 1128-40

32713 Ramos, H.C. et al. (2004) Bacterial flagellins: mediators of pathogenicity and host 328 immune responses in mucosa. Trends Microbiol. 12, 509-17

32914 Rumbo, M. et al. (2006) Mucosal interplay among commensal and pathogenic bacteria: 330 lessons from flagellin and Toll-like receptor 5. FEBS Lett. 580, 2976-84

$331 \quad 15$ Huh, J.-W. et al. (2014) UNC93B1 is essential for the plasma membrane localization and 332 signaling of Toll-like receptor 5. Proc. Natl. Acad. Sci. 111, 7072-7

33316 Didierlaurent, A. et al. (2004) Flagellin promotes myeloid differentiation factor 88334 dependent development of Th2-type response. J. Immunol. 172, 6922-30

33517 Gay, N.J. et al. (2014) Assembly and localization of Toll-like receptor signalling 336 complexes. Nat. Rev. Immunol. 14, 546-58

33718 Van Maele, L. et al. (2014) Airway structural cells regulate TLR5-mediated mucosal 338 adjuvant activity. Mucosal Immunol. 7, 489-500

33919 Van Maele, L. et al. (2010) TLR5 signaling stimulates the innate production of IL-17 and IL-22 by $\mathrm{CD} 3{ }^{\text {neg }} \mathrm{CD} 127^{+}$immune cells in spleen and mucosa. J. Immunol. $185,1177-85$

34120 Fougeron, D. et al. (2015) Indirect Toll-like receptor 5-mediated activation of 342 conventional dendritic cells promotes the mucosal adjuvant activity of flagellin in the 343 respiratory tract. Vaccine $33,3331-41$

34421 Morris, A.E. et al. (2009) Role of Toll-like receptor 5 in the innate immune response to 345 acute P. aeruginosa pneumonia. Am. J. Physiol. Lung Cell. Mol. Physiol. 297, L1112-9

34622 Vijay-Kumar, M. et al. (2008) Toll-Like Receptor 5-Deficient Mice Have Dysregulated 
Intestinal Gene Expression and Nonspecific Resistance to Salmonella-Induced TyphoidLike Disease. Infect. Immun. 76, 1276-81

34923 Andersen-Nissen, E. et al. (2007) Cutting edge: Tlr $5^{-/}$mice are more susceptible to $350 \quad$ Escherichia coli urinary tract infection. J. Immunol. 178, 4717-20

35124 Mathur, R. et al. (2012) A mouse model of Salmonella typhi infection. Cell 151, 590-602

35225 Zhang, D. et al. (2004) A toll-like receptor that prevents infection by uropathogenic $353 \quad$ bacteria. Science 303, 1522-6

35426 Mathur, R. et al. (2016) Mice Lacking TLR11 Exhibit Variable Salmonella typhi $355 \quad$ Susceptibility. Cell 164, 829-30

35627 Roach, J.C. et al. (2005) The evolution of vertebrate Toll-like receptors. Proc. Natl. Acad. $357 \quad$ Sci. $102,9577-82$

35828 Song, J. et al. (2016) Absence of TLR11 in Mice Does Not Confer Susceptibility to $359 \quad$ Salmonella typhi. Cell 164, 827-8

36029 Zhao, Y. and Shao, F. (2015) The NAIP-NLRC4 inflammasome in innate immune 361 detection of bacterial flagellin and type III secretion apparatus. Immunol. Rev. 265, 85$362 \quad 102$

36330 Gewirtz, A.T. et al. (2001) Cutting edge: Bacterial flagellin activates basolaterally 364 expressed TLR5 to induce epithelial proinflammatory gene expression. J. Immunol. 167, $365 \quad 1882-5$

36631 Adamo, R. et al. (2004) Pseudomonas aeruginosa Flagella Activate Airway Epithelial 367 Cells through asialoGM1 and Toll-Like Receptor 2 as well as Toll-Like Receptor 5. Am. $368 \quad$ J. Respir. Cell Mol. Biol. 30, 627-34

36932 Chabot, S. et al. (2006) TLRs Regulate the Gatekeeping Functions of the Intestinal 
Follicle-Associated Epithelium. J. Immunol. 176, 4275-83

37133 Schüller, S. et al. (2009) The ex vivo response of human intestinal mucosa to 372 enteropathogenic Escherichia coli infection. Cell. Microbiol. 11, 521-30

37334 Cario, E. and Podolsky, D.K. (2000) Differential alteration in intestinal epithelial cell 374 expression of toll-like receptor 3 (TLR3) and TLR4 in inflammatory bowel disease. Infect. $375 \quad$ Immun. $68,7010-7$

37635 Anas, A.A. et al. (2016) Lung epithelial MyD88 drives early pulmonary clearance of 377 Pseudomonas aeruginosa by a flagellin dependent mechanism. Am. J. Physiol. - Lung $378 \quad$ Cell. Mol. Physiol. 56, L219-28

37936 Janot, L. et al. (2009) Radioresistant cells expressing TLR5 control the respiratory 380 epithelium's innate immune responses to flagellin. Eur. J. Immunol. 39, 1587-96

38137 Chabot, S.M. et al. (2008) Effects of flagellin on the functions of follicle-associated 382 epithelium. J. Infect. Dis. 198, 907-10

38338 Sierro, F. et al. (2001) Flagellin stimulation of intestinal epithelial cells triggers CCL20384 mediated migration of dendritic cells. Proc. Natl. Acad. Sci. U. S. A. 98, 13722-7

38539 Sichien, D. et al. (2017) Development of conventional dendritic cells: from common bone 386 marrow progenitors to multiple subsets in peripheral tissues. Mucosal Immunol. 10, 831$387 \quad 44$

38840 Granot, T. et al. (2017) Dendritic Cells Display Subset and Tissue-Specific Maturation 389 Dynamics over Human Life. Immunity 46, 504-15

39041 Mizel, S.B. and Bates, J.T. (2010) Flagellin as an adjuvant: cellular mechanisms and $391 \quad$ potential. J. Immunol. $185,5677-82$

39242 Rumbo, M. et al. (2017) Flagellins as Adjuvants of Vaccines. In Immunopotentiators in 
Modern Vaccines (2nd edn) (Virgil E.J.C. Schijns and Derek T. O’Hagan, eds), pp. 129147, Elsevier

39543 He, B. et al. (2007) Intestinal Bacteria Trigger T Cell-Independent Immunoglobulin A2 396 Class Switching by Inducing Epithelial-Cell Secretion of the Cytokine APRIL. Immunity $26,812-26$

39844 Wilson, R.H. et al. (2012) The Toll-like receptor 5 ligand flagellin promotes asthma by priming allergic responses to indoor allergens. Nat. Med. 18, 1705-10

40045 Shim, J.-U. et al. (2016) Flagellin suppresses experimental asthma by generating 401 regulatory dendritic cells and T cells. J. Allergy Clin. Immunol. 137, 426-35

40246 McSorley, S.J. et al. (2002) Bacterial flagellin is an effective adjuvant for CD4+ T cells in 403 vivo. J. Immunol. 169, 3914-9

40447 Bobat, S. et al. (2011) Soluble flagellin, FliC, induces an Ag-specific Th2 response, yet 405 promotes T-bet-regulated Th1 clearance of Salmonella typhimurium infection. Eur. J. $406 \quad$ Immunol. 41, 1606-18

40748 Flores-Langarica, A. et al. (2015) Soluble flagellin coimmunization attenuates Th1 408 priming to Salmonella and clearance by modulating dendritic cell activation and cytokine production. Eur. J. Immunol. 45, 2299-2311

41049 Didierlaurent, A. et al. (2004) Flagellin promotes myeloid differentiation factor 88411 dependent development of Th2-type response. J. Immunol. 172, 6922-30

41250 Means, T.K. et al. (2003) The Toll-like receptor 5 stimulus bacterial flagellin induces 413 maturation and chemokine production in human dendritic cells. J. Immunol. 170, 5165-75

41451 Uematsu, S. et al. (2008) Regulation of humoral and cellular gut immunity by lamina 415 propria dendritic cells expressing Toll-like receptor 5. Nat. Immunol. 9, 769-76 
41652 Uematsu, S. et al. (2006) Detection of pathogenic intestinal bacteria by Toll-like receptor 4175 on intestinal CD11c+ lamina propria cells. Nat. Immunol. 7, 868-74

41853 Liu, H. et al. (2016) TLR5 mediates CD172 $\alpha(+)$ intestinal lamina propria dendritic cell 419 induction of Th17 cells. Sci. Rep. 6, 22040

42054 Kinnebrew, M. et al. (2012) Interleukin 23 Production by Intestinal CD103+CD11b+ 421 Dendritic Cells in Response to Bacterial Flagellin Enhances Mucosal Innate Immune 422 Defense. Immunity 36, 276-87

42355 Van Maele, L. et al. (2014) Activation of Type 3 Innate Lymphoid Cells and Interleukin 42422 Secretion in the Lungs During Streptococcus pneumoniae Infection. J. Infect. Dis. 210, $493-503$

42656 Flores-Langarica, A. et al. (2012) Systemic flagellin immunization stimulates mucosal CD103+ dendritic cells and drives Foxp3+ regulatory $\mathrm{T}$ cell and IgA responses in the mesenteric lymph node. J. Immunol. 189, 5745-54

42957 Lee, S.-J. et al. (2017) Dual Immunization with SseB/Flagellin Provides Enhanced $430 \quad$ Protection against Salmonella Infection Mediated by Circulating Memory Cells. $J$. Immunol. 199, 1353-61

43258 Taylor, D.N. et al. (2012) Development of VAX128, a recombinant hemagglutinin (HA) 433 influenza-flagellin fusion vaccine with improved safety and immune response. Vaccine $434 \quad 30,5761-9$

43559 Kim, J.R. et al. (2015) Inclusion of Flagellin during Vaccination against Influenza 436 Enhances Recall Responses in Nonhuman Primate Neonates. J. Virol. 89, 7291-7303

43760 Vijay-Kumar, M. et al. (2010) TLR5 or NLRC4 is necessary and sufficient for promotion 438 of humoral immunity by flagellin. Eur. J. Immunol. 40, 3528-34 
43961 Sanders, C.J. et al. (2009) Induction of adaptive immunity by flagellin does not require $440 \quad$ robust activation of innate immunity. Eur. J. Immunol. 39, 359-71

44162 Atif, S.M. et al. (2014) CD103-CD11b+ dendritic cells regulate the sensitivity of CD4 T442 cell responses to bacterial flagellin. Mucosal Immunol. 7, 68-77

44363 Vijay-Kumar, M. et al. (2008) Flagellin Treatment Protects against Chemicals, Bacteria, $444 \quad$ Viruses, and Radiation. J. Immunol. 180, 8280-5

44564 Yu, F. et al. (2010) Flagellin stimulates protective lung mucosal immunity: role of 446 cathelicidin-related antimicrobial peptide. J. Immunol. 185, 1142-9

44765 Zgair, A.K. (2012) Flagellin administration protects respiratory tract from Burkholderia $448 \quad$ cepacia Infection. J. Microbiol. Biotechnol. 22, 907-16

44966 Porte, R. et al. (2017) Flagellin-Mediated Protection against Intestinal Yersinia 450 pseudotuberculosis Infection Does Not Require Interleukin-22. Infect. Immun. 85, $451 \quad \mathrm{e} 00806-16$

45267 Kinnebrew, M.A. et al. (2010) Bacterial Flagellin Stimulates Toll-Like Receptor 5453 Dependent Defense against Vancomycin-Resistant Enterococcus Infection. J. Infect. Dis. $201,534-43$

45568 Jarchum, I. et al. (2011) Toll-like receptor 5 stimulation protects mice from acute $456 \quad$ Clostridium difficile colitis. Infect Immun 79, 1498-1503

45769 Muñoz, N. et al. (2010) Mucosal administration of flagellin protects mice from $458 \quad$ Streptococcus pneumoniae lung infection. Infect. Immun. 78, 4226-33

45970 Ogushi, K.I. et al. (2001) Salmonella enteritidis FliC (Flagella Filament Protein) Induces 460 Human $\beta$-Defensin-2 mRNA Production by Caco-2 Cells. J. Biol. Chem. 276, 30521-6

46171 Scharf, S. et al. (2010) Induction of human beta-defensin-2 in pulmonary epithelial cells 
by Legionella pneumophila: involvement of TLR2 and TLR5, p38 MAPK, JNK, NFkappaB, and AP-1. Am J Physiol Lung Cell Mol Physiol 298, L687-95

46472 Takahashi, A. et al. (2001) Production of beta-defensin-2 by human colonic epithelial 465 cells induced by Salmonella enteritidis flagella filament structural protein. FEBS Lett. $508,484-8$

46773 Abtin Arby, Eckhart Leopold, Mildner Michael, Gruber Florian, SchroderJens-Michael, 468 T.E. (2008) Flagellin is the principal inducer of the antimicrobial peptide S100A7c (psoriasin) in human epidermal keratinocytes exposed to Escherichia coli. FASEB J 22, $2168-76$

47174 Porte, R. et al. (2015) A Toll-Like Receptor 5 Agonist Improves the Efficacy of 472 Antibiotics in Treatment of Primary and Influenza Virus-Associated Pneumococcal 473 Mouse Infections. Antimicrob. Agents Chemother. 59, 6064-72

47475 Hossain, M.S. et al. (2014) Recombinant TLR5 agonist CBLB502 promotes NK cell475 mediated anti-CMV immunity in mice. PLoS One 9, e96165

47676 Zhang, B. et al. (2014) Prevention and cure of rotavirus infection via TLR5/NLRC4477 mediated production of IL-22 and IL-18. Science 346, 861-5

47877 Burdelya, L.G. et al. (2008) An Agonist of Toll-Like Receptor 5 Has Radioprotective 479 Activity in Mouse and Primate Models. Science 320, 226-30

48078 Burdelya, L.G. et al. (2013) Central role of liver in anticancer and radioprotective 481 activities of Toll-like receptor 5 agonist. Proc. Natl. Acad. Sci. 110, E1857-66

48279 Sfondrini, L. et al. (2006) Antitumor activity of the TLR-5 ligand flagellin in mouse 483 models of cancer. J. Immunol. 176, 6624-30

48480 Leigh, N.D. et al. (2014) A Flagellin-Derived Toll-Like Receptor 5 Agonist Stimulates 
Cytotoxic Lymphocyte-Mediated Tumor Immunity. PLoS One 9, e85587

$486 \quad 8$

487

488

489

490

491

492

493

494

495

Geng, D. et al. (2015) TLR5 ligand-secreting T cells reshape the tumor microenvironment and enhance antitumor activity. Cancer Res. 75, 1959-1971

82 Zheng, J.H. et al. (2017) Two-step enhanced cancer immunotherapy with engineered Salmonella typhimurium secreting heterologous flagellin. Sci. Transl. Med. 9, eaak9537

83 Messier-Solek, C. et al. (2010) Highly diversified innate receptor systems and new forms of animal immunity. Semin. Immunol. 22, 39-47

84 Tsujita, T. et al. (2006) Fish soluble Toll-like receptor (TLR)5 amplifies human TLR5 response via physical binding to flagellin. Vaccine 24, 2193-9

85 Smith, S.A. et al. (2012) Adaptive evolution of Toll-like receptor 5 in domesticated mammals. BMC Evol. Biol. 12, 122

86 Voogdt, C.G. et al. (2016) Reptile Toll-like receptor 5 unveils adaptive evolution of bacterial flagellin recognition. Sci Rep 6, 19046

87 Schroder, K. and Bosch, T.C.G. (2016) The origin of mucosal immunity: Lessons from the holobiont Hydra. MBio 7, 1-9

88 Chinchilla, D. et al. (2007) A flagellin-induced complex of the receptor FLS2 and BAK1 initiates plant defence. Nature 448, 497-500

89 Hawn, T.R. et al. (2003) A common dominant TLR5 stop codon polymorphism abolishes flagellin signaling and is associated with susceptibility to legionnaires' disease. J. Exp. Med. 198, 1563-72

Crispo, M. et al. (2013) Transgenic mouse model harboring the transcriptional fusion Cc120-luciferase as a novel reporter of pro-inflammatory response. PLoS One 8, e 78447

Hawn, T.R. et al. (2009) Toll-like receptor polymorphisms and susceptibility to urinary 
tract infections in adult women. PLoS One 4, e5990

50992 Grossman, S.R. et al. (2013) Identifying recent adaptations in large-scale genomic data.

$510 \quad$ Cell $152,703-13$

51193 Meena, N.K. et al. (2015) Association of TLR5 Gene Polymorphisms in Ulcerative Colitis

512 Patients of North India and Their Role in Cytokine Homeostasis. PLoS One 10, e0120697

51394 Hawn, T.R. et al. (2005) A stop codon polymorphism of Toll-like receptor 5 is associated

514 with resistance to systemic lupus erythematosus. Proc. Natl. Acad. Sci. U. S. A. 102, $515 \quad 10593-7$

51695 Gewirtz, A.T. et al. (2006) Dominant-negative TLR5 polymorphism reduces adaptive

517 immune response to flagellin and negatively associates with Crohn's disease. AJP $518 \quad$ Gastrointest. Liver Physiol. 290, G1157-63

51996 Vijay-Kumar, M. et al. (2007) Deletion of TLR5 results in spontaneous colitis in mice. $J$. $520 \quad$ Clin. Invest. 117, 3909-21

52197 Chassaing, B. et al. (2014) Intestinal Epithelial Cell Toll-like Receptor 5 Regulates the 522 Intestinal Microbiota to Prevent Low-Grade Inflammation and Metabolic Syndrome in $523 \quad$ Mice. Gastroenterology 147, 1363-77

52498 Singh, V. et al. (2015) Microbiota-Dependent Hepatic Lipogenesis Mediated by Stearoyl 525 CoA Desaturase 1 (SCD1) Promotes Metabolic Syndrome in TLR5-Deficient Mice. Cell 526 Metab. 22, 983-996

52799 Etienne-Mesmin, L. et al. (2016) Hepatocyte Toll-Like Receptor 5 Promotes Bacterial 528 Clearance and Protects Mice Against High-Fat Diet-Induced Liver Disease. Cell Mol 529 Gastroenterol Hepatol 2, 584-604

530100 Okumura, R. et al. (2016) Lypd8 promotes the segregation of flagellated microbiota and 
colonic epithelia. Nature 532, 117-21

532101 Oh, J.Z. et al. (2014) TLR5-mediated sensing of gut microbiota is necessary for antibody responses to seasonal influenza vaccination. Immunity 41, 478-92

534102 Cullender, T.C. et al. (2013) Innate and adaptive immunity interact to quench microbiome flagellar motility in the gut. Cell Host Microbe 14, 571-81

536103 Kofoed, E.M. and Vance, R.E. (2011) Innate immune recognition of bacterial ligands by NAIPs determines inflammasome specificity. Nature 477, 592-5

538104 Kortmann, J. et al. (2015) Cutting Edge: Inflammasome Activation in Primary Human Macrophages Is Dependent on Flagellin. J. Immunol. 195, 815-9

540105 Lin, K.-H. et al. (2016) Carboxyl-terminal fusion of E7 into Flagellin shifts TLR5 541 activation to NLRC4/NAIP5 activation and induces TLR5-independent anti-tumor 542 immunity. Sci. Rep. 6, 24199 


\section{Figure Legends}

547 Figure 1. Structure of flagellin and molecular mechanisms of TLR5-induced immunity. (A)

548 Organization of flagellum and flagellin. Motile bacteria produce flagella composed mainly of 549 polymerized flagellin. Salmonella enterica serovar Typhimurium flagellin consists of 4 domains:

550 the terminal $\alpha$-helixes (D0), the central $\alpha$-helixes (D1), and the hypervariable $\beta$-sheets and turns

551 (D2 and D3). The $\alpha$-helixes regions (D0+D1) are required for filament architecture and motility

552 functions and are embedded in the filament core. When released from the flagellum, the structure

553 of D0 domain of flagellin is disordered. The exposed D0 and D1 domains on monomeric

554 flagellin are mandatory for immune signaling. (B) The detection of flagellin by TLR5 triggers

555 signaling cascade involving the universal TLR-specific adapter molecule MyD88, and

556 downstream regulators such as NF- $\kappa$ B, MAPK, thereby activating transcription of genes

557 involved in innate and adaptive immunity. (C) Schematic view of TLR5-flagellin structure. The

558 interactions of the D1 domain of flagellin with TLR5 at the cell surface is highlighted. This view

559 is based on the protein databank structure $3 \mathrm{v} 47$.

561 Figure 2: Response of epithelial cells to flagellin. The epithelium forms the interface between

562 the internal and the external environment. Epithelial cells of the mucosa are the main sentinel 563 cells that sense bacterial flagellin and stimulates TLR5 signaling. The epithelial cell-driven

564 immediate innate response is characterized by (1) secretion of various anti-microbial factors that

565 limit bacterial growth, and (2) production of pro-inflammatory cytokines and chemokines that

566 recruit phagocytes, mainly the circulating neutrophils and monocytes. Epithelial cells also have a 567 significant imprint on adaptive immunity by activating immature tissue-resident cDCs. Mature 
568 cDCs later migrate through afferent lymphatic vessels into the lymph nodes where they stimulate

569 differentiation of $\mathrm{CD}^{+}$lymphocytes into Th1 and Th2 cells as well as B lymphocytes into 570 antibody-producing plasma cells, mainly IgA and IgG-producing cells. Polymeric

571 immunoglobulin receptor ( $\mathrm{pIgR}$ ) that is expressed on the basolateral side of epithelial cells, binds

572 to dimeric IgA and is transported to the apical side where it gets cleaved to release secretory IgA

573 (SIgA). The crosstalk between epithelial cells and DCs shape the adaptive arm of the immune 574 response.

575

Figure 3. Direct activation of tissue-resident dendritic cells: from type 3 innate lymphoid

577 cells to conventional lymphocytes. Flagellin can directly activate sentinel cells present in 578 mucosal tissues, a condition that appears when motile pathogenic bacteria breach epithelium.

579 The dark-shaded cells represent damaged epithelium. The tissue-resident conventional dendritic 580 cells $(\mathrm{cDCs})$ are key players in the process of detection and integration of danger signals. Thus, 581 TLR5 signaling in cDCs induces the secretion of cytokines secretion including IL-12p40, IL-23 582 (IL-12p40 + IL-23p19) and IL-6 that are essential in the differentiation/activation of lymphoid 583 cells, the expression of co-stimulatory molecules such as CD80 and CD86 and the upregulation 584 of antigen presentation. The immediate consequences are the swift activation of mucosal type 3 585 innate lymphoid cells (ILC3) into IL-22 producing cells. Notably, IL-22R is only expressed by 586 structural cells, especially ECs that, in turn, switch on a program dedicated to microbial 587 clearance and tissue repair to promote recovery and homeostasis. Flagellin-activated ILC3 might 588 represent essential effectors for protection of mucosa against invasive pathogenic bacteria. The 589 mid-term effect of $\mathrm{cDC}$ maturation is the priming of naïve $\mathrm{T}$ and $\mathrm{B}$ lymphocytes into effector 590 cells. There is evidence that flagellin-mediated signaling in cDC stimulates the differentiation 
591 into Th1, Th2 and Th17 $\mathrm{CD}^{+}$Th cells and elicits a prominent systemic IgG1 response and local

592 IgA response. Such an adaptive response might help long-term protection against pathogens. 
595 BOX 1

\section{Evolution TLR5 and flagellin recognition.}

597 Genome sequence analysis of a large number of animal phyla shows that TLRs are present in 598 virtually all eumetazoans, indicating that TLRs are ancient PRMs [83]. Concerning TLR5, 599 orthologues of this receptor are found in most vertebrates, i.e., mammals, birds, reptiles, 600 amphibians and fishes [84-86]. The extracellular LRRs of TLR5 orthologues are responsible for 601 flagellin recognition as inferred from structure and sequence analysis performed with zebrafish 602 TLR5 [9]. Interestingly, the most divergent sequences are found in fish with several species 603 encoding a soluble form of TLR5 formed by LRRs. The function of soluble TLR5 is 604 controversial with studies that suggest enhancement of NF- $\kappa \mathrm{B}$ signaling in cells expressing 605 membrane-bound TLR5 or inhibition of cell activation by sequestration [84].

606 The genome of primitive metazoan hydra that emerged more than 500 million years ago, 607 encodes, instead of the typical TLRs, four transmembrane proteins: two proteins constituted by 608 TIR domains and short extracellular regions (HyTRR1 and HyTRR2), and two proteins with 609 LRR-containing ectodomains and short cytoplasmic tails (HyLRR1 and HyLRR2) [87]. 610 HyLRR2 with HyTRRs transduce activation signals upon flagellin stimulation. Thus, hydra ECs 611 produce antimicrobial peptide expression, which in turn may modulate the microbiota associated 612 with the endodermal layer.

613 Plants have also developed a unique TLR-like FLS2 to detect flagellin of pathogenic 614 phytobacteria [88]. This process was also related to defense mechanisms to promote integrity of 
615 plant cells upon infection. In conclusion, environmental monitoring of flagellin and the shaping

616 of host-associated defense mechanisms were conserved during evolution.

619 BOX 2

\section{TLR5 polymorphism in infection and chronic diseases}

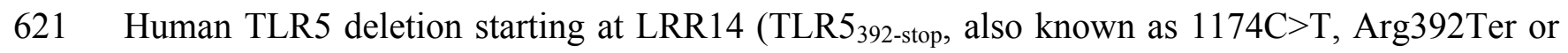
622 rs5744168) is associated with the loss of responsiveness to flagellin [89]. The variant TLR5 392 -

623 stop is dominant negative and is present in about 5\% in the global human populations $(2.3 \%$ in 624 African, 3.0\% in American, 3.9\% in East Asian, 6.1\% in European, and 10.3\% in South Asian 625 individuals; www.ensembl.org/Homo_sapiens/Variation/Population?db=core;r=1:223111358$626223112358 ; \mathrm{v}=\mathrm{rs} 5744168 ; \mathrm{vdb}=$ variation; $\mathrm{vf}=3234911)$. Remarkably, a similar mutation in the

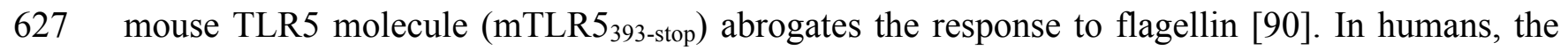
628 TLR5 392 -stop mutation is associated with susceptibility to lung infections with Legionella 629 pneumophila [89], and to urinary tract infections with uropathogenic E. coli [91]. Whether other 630 polymorphisms associated with a reduced TLR5 signaling impact on diseases remains to be 631 defined [92]. Moreover, similar vulnerability is found in TLR5-deficient animals, highlighting a 632 major contribution of TLR5 to innate antimicrobial defenses.

633 Besides, TLR5 polymorphism is also involved in various chronic diseases. In humans, TLR5

634 deficiency and TLR5 hypomorphic variants increase the risk of ulcerative colitis but promotes 635 resistance to systemic lupus erythematosus or Crohn's disease [93-95]. In mice, TLR5 deficiency 636 is associated with changes in microbiota that in turn contribute to spontaneous colitis and 637 metabolic syndrome [60,96]. Loss of intestinal epithelial TLR5 seems to alter the microbiota 
638 composition and trigger low-grade inflammation and metabolic abnormalities [97]. Furthermore,

639 the changes of short-chain fatty acids' composition due to TLR5-associated dysbiosis promotes

640 hepatic lipogenesis [98] whereas hepatic TLR5 deficiency increases the hepatic abnormalities

641 induced by a high-fat diet [99]. Recently, the Ly6/ PLAUR domain containing 8 (Lypd8) was

642 found to interact with flagella and regulate the segregation of motile bacteria within gut lumen

643 [100]. Unraveling how Lypd8 and TLR5 complement, synergize or antagonize activity on

644 immune signaling to flagellated bacteria is a major issue for future investigations.

645 TLR5 signaling can impact the response to a vaccine. In humans, TLR5 expression positively

646 correlates with response to vaccination [101]. TLR5-deficient mice have impaired antibody

647 response after flu vaccination compared to wild-type animals. This effect is related to deficient

648 TLR5 signaling in macrophages and short-lived plasma cells. Besides, Cullender et al. showed

649 that flagellin-specific antibodies (and TLR5 signaling) are essential to regulate populations of

650 flagellated bacteria from microbiota, thus having a global impact on immune responses [102].

651 Consistent with these findings, germ-free mice have impaired antibody response to a vaccine that

652 can be rescued by colonization with flagellated bacteria [101].

655 BOX 3

656 NLRC4-dependent inflammasome activation

657 Bacterial flagellin is a unique MAMP, in the sense that it can trigger immune signaling in the 658 extracellular medium via TLR5 as well as in the cytoplasmic compartment. Thus, in mouse 659 phagocytes, the cytosolic detection of flagellin by PRM of the NAIP family (for nucleotide660 binding and oligomerization domain-like receptor (NLR) apoptosis-inhibitory protein) promotes 
661 the activation of inflammasome through the recruitment and the oligomerization of NLRC4

662 (NLR family caspase recruitment domain-containing 4) (reviewed in [29]) (Figure I). This 663 gatekeeping mechanism provides alternative sensing for intracellular flagellated bacteria or 664 intracellular injection of flagellin by bacterial secretion systems. Inflammasome activation is 665 associated with caspase 1-dependent maturation of the pro-inflammatory cytokines IL-1 $\beta$ and 666 IL-18 and with pyroptosis, a cell death linked to cytokine release. Thus, NAIP/NLRC4 activation 667 impacts the intracellular replication of flagellated bacteria and participates to host's defense. In 668 mice, the PRM NAIP5 and NAIP6 bind flagellin carboxy-terminal amino acids [103]. In 669 contrast, NAIP2 activates NLRC4-dependent inflammasome by sensing needle components of 670 type 3 secretion systems from gram-negative bacteria $[29,103]$.

671 Humans produce a unique NAIP molecule, but flagellin detection by this sensor remains 672 controversial $[29,104]$. Remarkably, in most studies using flagellin to manipulate immune 673 responses in animal models or clinical trials, formulations are made of soluble monomer that is 674 poorly capable per se of entering mammalian cells. Further studies are required to determine 675 whether flagellin activate the inflammasome machinery in humans as it does in mice and how 676 relevant it is in immunity.

677 NLRC4 activation can significantly hamper the TLR5-mediated adjuvant activity of flagellin 678 [60]. Adaptive immune responses raised against a flagellin-antigen protein is influenced by the 679 site of fusion ( $\mathrm{N}$ or $\mathrm{C}$ terminus of flagellin), an effect that is dependent on differential TLR5 and 680 NLRC4 signaling [105]. Additional investigations are needed to characterize the molecular 681 determinant of flagellin and antigen that might control TLR5 and NLRC4 signaling pathways. 682 
683 Figure I: Schematic view of flagellin-mediated inflammasome activation. Upon detection of

684 cytosolic flagellin by NAIP5 or NAIP6, NLRC4 is recruited and oligomerizes. This process is 685 dependent on the apoptosis-associated speck-like protein containing a CARD (ASC) and results 686 in the proteolytic cleavage of the pro-caspase 1 into active caspase 1 that in turn mediates 687 inflammatory responses via interleukin activation. 
A

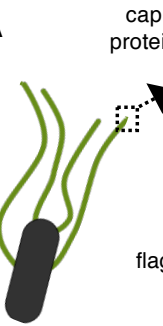

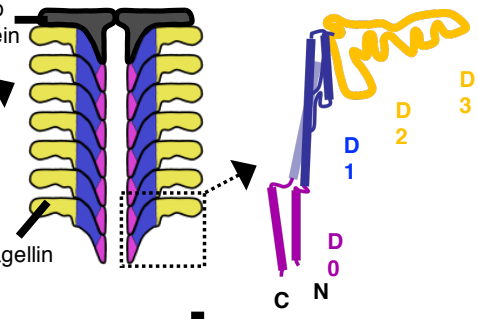

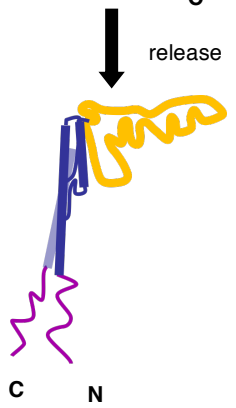

C

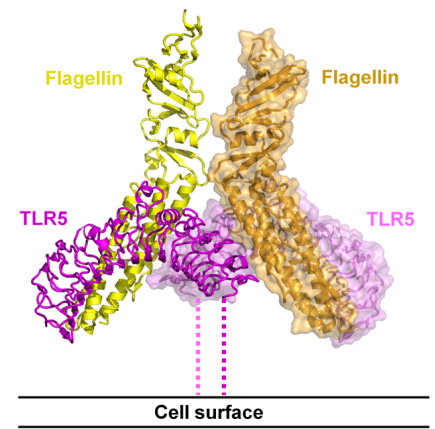

B

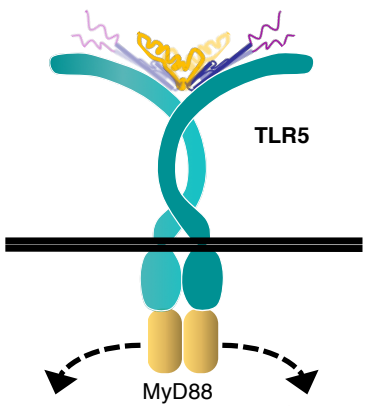

MAPK activation

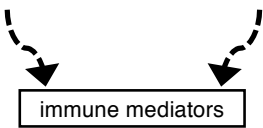

Figure 1 


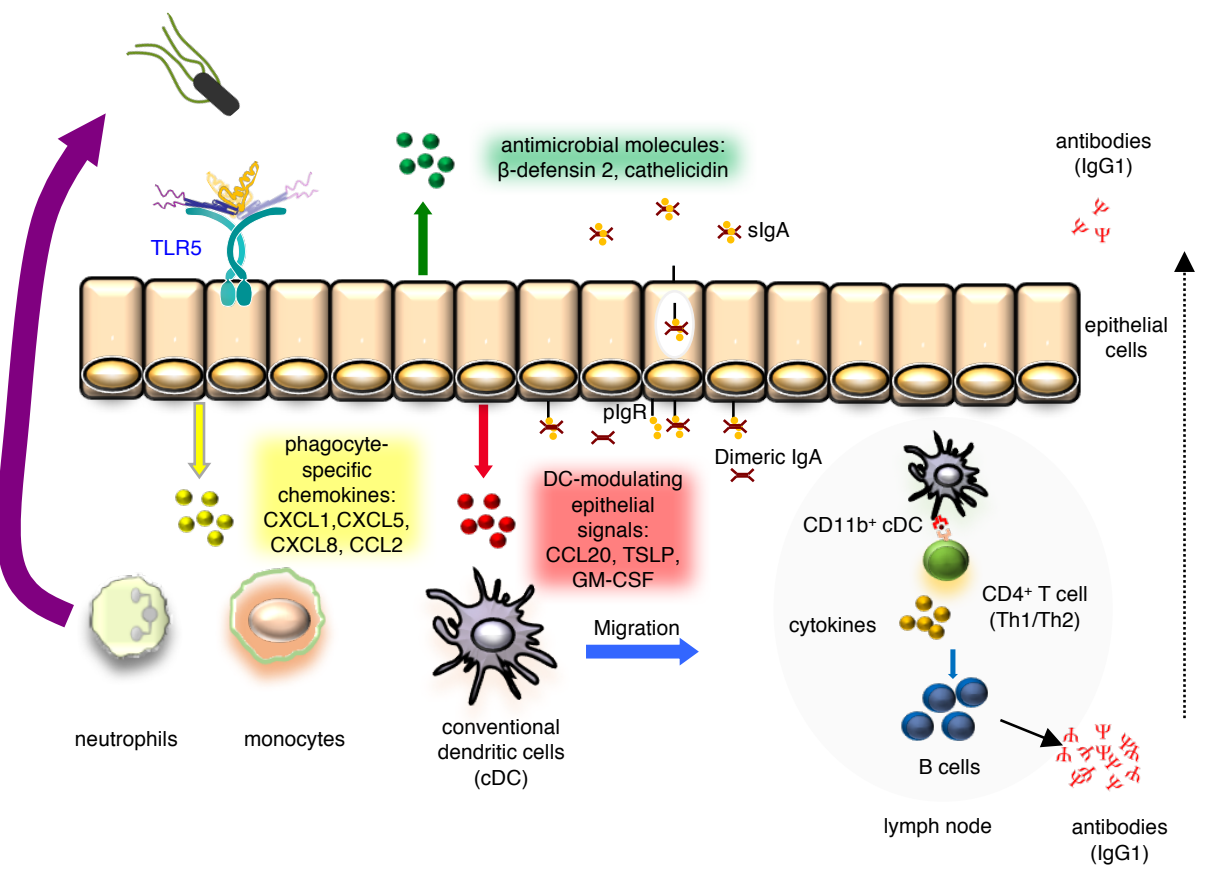

Figure 2 
